\title{
Burnout y Work Engagement en Docentes Universitarios de Zacatecas
}

\author{
BURNOUT AND WORK ENGAGEMENT IN ZACATECAS UNIVERSITY TEACHERS
}

\author{
Dellanira Ruiz de Chávez Ramírez ${ }^{1}$, Manuel Pando Moreno², Carolina Aranda Beltrán ${ }^{3}$, Cristina Almeida Perales ${ }^{4}$ \\ 1. Doctor en Ciencias de la Salud en el Trabajo. Maestria en Ciencias de la Salud. Universidad Autónoma de Zacatecas. \\ 2. Doctor en Sociología y Metodología de las Ciencias. Universidad de Guadalajara. \\ 3. Doctor en Ciencias de la Salud Pública. Universidad de Guadalajara. \\ 4. Maestra en Ciencias. Maestría en Ciencias de la Salud. Universidad Autónoma de Zacatecas.
}

\section{RESUMEN}

Objetivo: Determinar la relación que existe entre el Síndrome de Burnout y el Work Engagment con variables demográficas y laborales en docentes universitarios de Zacatecas. Material y métodos: a través de una metodología cuantitativa se diseñó un estudio de tipo observacional, analítico, transversal en una muestra de 98 mujeres y 58 hombres. Se utilizó el instrumento de Maslach Burnout Inventory y el Utrecht Work Engagement Scale de 17 reactivos. Resultados: $62,8 \%$ mujeres y $37,2 \%$ hombres con una edad promedio de 43,9 años; respecto al Burnout se evidenció un alto nivel de agotamiento y en el 35,3\% con al menos una dimensión quemada. Se encontró una relación $\left(p_{-} 0,05\right)$ con el sexo, la edad y el grado académico con el agotamiento emocional. En cuanto al Work Engagement se encontraron altos niveles en el 99,4\% de los participantes. Conclusión: Lo anterior demuestra que, a pesar del desgaste físico y psicológico en el que se encuentra el docente ante los constantes cambios, cuenta con altos niveles de compromiso laboral.

(Ruiz de Chávez D, Pando M, Aranda C, Almeida C, 2014. Burnout y Work Engagement en Docentes Universitarios de Zacatecas. Cienc Trab. May-Ago; 16 [50]: 116-120).

Palabras Clave: DOCENTES, BURNOUT, ENGAGEMENT.

\section{ABSTRACT}

Objective: This study explored the relationship between the Burnout Syndrome and Work Engagement with demographic and labor variables among university academics of Zacatecas. Materials and methods: through a quantitative methodology, an observational analytical, cross-type study was designed by using a sample size of 98 women and 58 men. The instrument of Maslach's Burnout Inventory and the Utrecht Work Engagement Scale with 17 items were used. Results: 62,8\% female and 37,2\% male had, on average, an age of 44 years. Regarding the Burnout, a high level of exhaustion was observed, where $35,3 \%$ of participants had, at least, one dimension burned. A significant relationship $\left(p_{-} 0,05\right)$ between sex, age, and gender with emotional exhaustion was found. The 99,4\% of the participants had high levels the Work Engagement. Conclusion: Notwithstanding the constant academic changes, and the physical and psychological strain found among academics, they presented high levels of job engagement.

Key words: ACADEMICS, BURNOUT, ENGAGEMENT.

\section{INTRODUCCIÓN}

Los desafíos de las universidades en el siglo XXI se caracterizan por hacer frente a múltiples cambios en los paradigmas del conocimiento y dichos cambios conllevan a transformaciones organizacionales que comprenden nuevas tareas en la actividad docente, representando un mayor estrés ya que dichas tareas no se limitan a las realizadas solamente dentro del aula sino que "obliga a la realización de varias tareas a la vez que van desde el manteni-

Correspondencia / Correspondence:

Dellanira Ruiz de Chávez Ramirez

Maestría en Ciencias de la Salud, Universidad Autónoma de Zacatecas, Campus Siglo XXI.

Carretera Zacatecas-Guadalajara Km 6 s/n Ejido la Escondida. Zacatecas. Teléfono: (52 492) 9256690 ext. 4526

e-mail: deyaruizdechavez@gmail.com

Recibido: 02 de Mayo de 2014 / Aceptado: 10 de Julio 2014 miento de la escuela, organización de planes y programas, planeación de actividades docentes, elaboración de materiales didácticos, realización de estadística escolar, entre otras" tal como lo señala Aldrete (2003). ${ }^{1}$ Entre las nuevas tareas en la educación superior están: integrar la investigación a partir de líneas de generación y aplicar el conocimiento con pertinencia y que dé solución a problemas del entorno con la formación; incrementar la calidad con perfiles deseables; institucionalizar el programa de tutorías; $y$, sistematizar el seguimiento de la trayectoria escolar, lo que impone a los docentes difíciles retos y competencias que sobrepasan las meramente didácticas y pedagógicas. En este sentido existe un deterioro de la salud física y mental que repercute en el compromiso y la satisfacción laboral (Sandoval 2005). ${ }^{2}$

Según Gómez (2000), " "nunca hasta ahora el docente había estado sometido a demandas tan complejas, intensas, además de contradictorias", por lo que el éxito o el fracaso de una reforma educativa depende de la forma en la cual los docentes interpretan, analizan e intervienen, teniendo como resultado la aceptación o el rechazo de cualquier cambio educativo. La forma en cómo responde el docente afecta importantemente su bienestar psicoló- 
gico en el trabajo, desarrollando procesos ansioso-depresivos como respuesta a un estrés prolongado mejor conocido como Sindrome de Burnout 4 (Cifre, Llorens, Salanova y Martinez 2003), el cual se presenta en aquellas personas que no fueron capaces de desempeñarse de manera adecuada ante tales cambios y exigencias (Schaufeli 2002; Maslach, Schaufeli y Leiter 2001)..$^{5,6}$

Lo anterior va conformando una realidad cada vez más compleja, la cual produce pobres expectativas de éxito con una sensación de impotencia o frustración que afecta la salud mental del docente, apareciendo el Síndrome de Burnout que ocurre en aquellas personas que no lograron adaptarse en una forma afectiva ni cognitiva ante una situación de estrés. Dicho síndrome consta básicamente de tres dimensiones que son: el agotamiento emocional, mostrando una sensación de no poder dar más apoyo emocional; la despersonalización, actitudes negativas hacia otros; y, la realización personal en el trabajo, caracterizada por sentimientos de baja competencia y pérdida de la autoestima (Maslach, Schaufeli y Leiter, 2001). ${ }^{6}$

Por otro lado, también existe el compromiso laboral denominado Work Engagement, definido como un estado psicológico positivo relacionado con el trabajo caracterizado por el vigor, dedicación y absorción (Schaufeli, 2002). ${ }^{5}$ Con este tipo de respuestas existen las personas que experimentan work engagement, quienes se sienten altamente competentes, motivadas y capaces de responder a las demandas del puesto de trabajo con absoluta eficacia. El Work Engagement o compromiso laboral aparece cuando se percibe un gran número de facilitadores, y hace que el trabajador se perciba como competente y capaz de desempeñar eficientemente cualquier cambio o innovación organizacional. El estar vinculados psicológicamente con el trabajo es mostrar conductas tales como altruismo organizacional, trabajo en equipo o altruismo laboral. El Work Engagement comienza a estudiarse como un constructo motivacional positivo relacionado con el trabajo y constituido por tres dimensiones según Schaufeli y Bakker $^{7}$ (2001): vigor identificado por altos niveles de energía y resistencia mental; la dedicación definida como, entusiasmo, inspiración y orgullo y la tercera dimensión conocida como absorción, que reconoce una alta concentración en la cual se siente que el tiempo pasa rápidamente por la máxima concentración que se han puesto en las tareas laborales.

En tal contexto laboral modificado por los cambios en los modelos educativos, con rediseños en los contenidos, procesos y estructuras se planteó la pregunta de investigación: ¿Qué factores demográficos y laborales tienen una importante relación con el Sindrome de Burnout y el Work Engagement en docentes universitarios de Zacatecas?

\section{MATERIAL Y MÉTODOS}

Se realizó un estudio hipotético-deductivo, no experimental, observacional, analítico, transversal en una muestra probabilística de 156 docentes mismos que se seleccionaron de manera aleatoria de las cinco Unidades Académicas que conforman el Área de Ciencias de las Unidades Académicas que conforman el Área de Ciencias de la Salud (Nutrición, Medicina, Químico Farmacobiólogo, Odontología y Enfermería) siempre y cuando cumplieran con los criterios de inclusión: docentes del Área de Ciencias de la Salud de medio tiempo y tiempo completo, con base o tiempo determinado. Que se encuentren laborando frente a grupo y que cuenten con más de 6 meses de antigüedad en la institución. Se aplicó un número mayor de cuestionarios con un 29,5\% en la Unidad Académica de Odontología debido a la alta población de docentes que en ella laboran.

Para evaluar la presencia del Síndrome de Burnout se utilizó el Maslach Burnout Inventory (MBI) en su versión traducida y validada al español ${ }^{8}$ (Gil Monte 2002) consta de 22 ítems con cinco opciones de respuesta (escala Likert de 0 a 4) que identifica el agotamiento emocional (AE), la despersonalización (D), y la realización personal en el trabajo (RPT). Las puntuaciones de cada escala se obtienen al sumar los valores de cada una de las dimensiones identificando niveles de alto, medio y bajo. Para la dimensión de $\mathrm{AE}$ y $\mathrm{D}$, si la puntuación es mayor indican un mayor grado de burnout; en cambio, para la RPT refleja mayores índices de burnout si la puntuación es menor. La prevalencia global del burnout se determina cuando los sujetos tienen la presencia de, al menos, dos dimensiones afectadas.

Para medir el Work Engagement se utilizó el Utrecht Work Engagement Scale (UWES), el cual contiene una escala de tipo likert con 7 opciones de respuesta que van de "nunca" a "todos los días" y 17 ítems los cuales deberán sumarse de acuerdo a cada dimensión para obtener una calificación; incluye reactivos para medir las tres dimensiones del constructo vigor, dedicación $y$ absorción. El instrumento cuenta con un alfa de Cronbach de 0,83, 0,92 y 0,82 respectivamente (Schaufeli, 2002). ${ }^{5}$ El análisis y procesamiento de los datos se realizó en el programa SPSS V.18 y se llevó a cabo un análisis descriptivo y analítico. La asociación de las variables se determinó a través de chi cuadrada con significancia estadística de $\mathrm{p}<0,05$ o un OR (factor de riesgo) mayor a $1 \mathrm{y}$ el intervalo de confianza no contiene a la unidad.

\section{RESULTADOS}

La población estudiada estuvo conformada por 98 mujeres y 58 hombres. En cuanto a las características sociodemográficas se consideraron solo el sexo, la edad, estado civil y el número de hijos. El 62,8\% de los docentes corresponde al sexo femenino, lo cual da cuenta de los factores culturales que marcan las condiciones, las formas y los medios de acceso de las mujeres en la educación pública en todos los niveles. Las edades oscilaron de los 24 a los 60 años con un promedio de 43,9 años y una desviación estándar de 9,57, lo que refiere una población relativamente joven.

El estado civil predominante fue el casado con un 64\% del total de los docentes. Al elaborar la recodificación de la variable de estado civil con pareja se encontró el 67,3\% y sin pareja el 32,7\%. Esto demuestra que la mayoría de los profesionales cuentan con pareja. En cuanto al grado académico se cuenta con más de la mitad de los docentes cualificados (con nivel de maestría un 48\% y con grado de doctor un 12,2\%), lo que nos indica que la institución cuenta con un 60,2\% de los docentes entrevistados con perfil deseable. La antigüedad se encontró con un mayor porcentaje: aquellos de más de 15 años fueron un 39,1\%; esto nos demuestra que para cubrir el criterio de inclusión de medio tiempo y tiempo completo como categoria laboral se requiere un mayor número de años laborando.

En la categoría laboral, el 70,5\% cuenta con tiempo completo dentro de la universidad, lo que refleja una mejor estabilidad laboral, pero con una categoría que les obliga a un mayor número 
de actividades como: docencia, investigación, tutorias, gestión, difusión y vinculación.

Respecto al Sindrome de Burnout se encontró que 74 docentes tuvieron un 47,4\% de agotamiento emocional (nivel alto con 23,7\%; nivel medio un 23,7\%), 42 docentes presentaron despersonalización en un 26,9\% (nivel alto 3,8\%; nivel medio 23,1\%) y solo el 7,7\% mostraron altos niveles en la falta de realización personal en el trabajo. En cuanto al número de dimensiones afectadas, el 36,5\% de los docentes se encontró sin ninguna dimensión quemada; con daño en una dimensión, el 35,3\%; dos dimensiones, el 22,4\%; y, con tres dimensiones, el 5,8\%, lo cual nos indica una prevalencia de 63,5\%. Al medir las dimensiones del Work Engagement se encontró que el 99\% de los docentes cuentan con altos niveles en las dimensiones de vigor, dedicación y absorción, lo que hace evidente un alto compromiso laboral.

Al revisar la relación que existe entre las variables sociodemográficas y laborales con el Síndrome de Burnout en su dimensión de agotamiento emocional, se encontró una asociación significativa entre éste y el sexo femenino, al contar con una edad menor a 45 años y tener el grado académico de doctor $(\mathrm{p} \leq 0,05)$. Respecto al OR (Odds Ratio) del agotamiento emocional con este tipo de

Tabla 1.

Relación de variables sociodemográficas y laborales con el agotamiento emocional.

\begin{tabular}{|c|c|c|c|c|c|}
\hline \multirow{2}{*}{$\begin{array}{l}\text { Variables Sociodemográficas } \\
\text { y Laborales }\end{array}$} & \multicolumn{5}{|c|}{ Agotamiento Emocional } \\
\hline & & chi & $p$ & OR & IC \\
\hline \multirow[t]{2}{*}{ Sexo } & Femenino Vs & 6,21 & 0,013 & 1,00 & \\
\hline & Masculino & & & 2,33 & $1,19-4,56$ \\
\hline \multirow[t]{2}{*}{ Edad } & Menos de 45 años Vs & 7,47 & 0,006 & 1,00 & \\
\hline & De 45 y más años & & & 2,43 & $1,28-4,64$ \\
\hline Estado & Sin pareja Vs & 1,69 & 0,193 & 1,00 & \\
\hline Civil & Con pareja & & & 0,64 & $0 ; 32-1,25$ \\
\hline Categoria & Hora/Clase Vs & 1,35 & 0,245 & 1,00 & \\
\hline Laboral & Otra Categoria & & & 1,68 & $0,69-4,05$ \\
\hline \multirow[t]{2}{*}{ Antigüedad } & Menos de 5 años Vs & 0,05 & 0,817 & 1,00 & \\
\hline & 5 años y más & & & 1,08 & $0,54-2,17$ \\
\hline Unidad & QFB Vs & 3,60 & 0,058 & 1,00 & \\
\hline Académica & Otra Unidad & & & 2,50 & $0,94-6,58$ \\
\hline Grado & Doctorado Vs & 5,97 & 0,014 & 1,00 & \\
\hline Académico & Otro grado & & & 3,59 & $1,22-10,53$ \\
\hline
\end{tabular}

Fuente: Directa

Tabla 2.

Relación de variables sociodemográficas y laborales con la despersonalización.

\begin{tabular}{|c|c|c|c|c|c|}
\hline \multirow{2}{*}{$\begin{array}{l}\text { Variables Sociodemográficas } \\
\text { y Laborales }\end{array}$} & \multicolumn{5}{|c|}{ Despersonalización } \\
\hline & & chi & $p$ & $\mathrm{OR}$ & IC \\
\hline \multirow[t]{2}{*}{ Sexo } & Masculino Vs & 2,68 & 0,102 & 1,00 & \\
\hline & Femenino & & & 1,81 & $0,88-3,73$ \\
\hline \multirow[t]{2}{*}{ Edad } & Menos de 45 años Vs & 2,37 & 0,123 & 1,00 & \\
\hline & de 45 y más años & & & 0,57 & $0,27-1,16$ \\
\hline Estado & Con pareja Vs & 0,62 & 0,732 & 1,00 & \\
\hline Civil & Sin pareja & & & 0,77 & $0,28-2,13$ \\
\hline Categoría & Medio Tiempo Vs & 0,00 & 0,968 & 1,00 & \\
\hline Laboral & Otra categoria & & & 1,02 & $0,37-2,81$ \\
\hline \multirow[t]{2}{*}{ Antigüedad } & Menos de 5 años Vs & 5.93 & 0,015 & 1,00 & \\
\hline & 5 años y más & & & 3,12 & $1,21-8.04$ \\
\hline Unidad & OFB Vs & 3,13 & 0,077 & 1,00 & \\
\hline Académica & Otra Unidad & & & 2,31 & $0,89-5.98$ \\
\hline Grado & Doctorado Vs & 4.59 & 0,032 & 1,00 & \\
\hline Académico & Otro grado & & & 2,83 & $1,06-7.57$ \\
\hline
\end{tabular}

Fuente: Directa variables (Tabla 1), se encontró dos veces mayor riesgo en mujeres, dos veces más riesgo en aquellos de menos de 45 años de edad y un riesgo de 3 veces más en personal académico con grado de doctor para presentar agotamiento emocional.

Para la despersonalización se encontró una asociación significativa con la antigüedad de menos de 5 años (Tabla 2), en quienes existe un riesgo de tres veces más para presentar despersonalización. En cuanto al grado académico, también se observa una asociación significativa ( $p \leq 0,05$ ), por lo que cualquier otro grado tiene menor riesgo de presentar esta dimensión del Burnout que quienes tienen doctorado. Esto se puede atribuir a que a mayor preparación académica el docente cuenta con una mayor carga de trabajo, desgaste físico y emocional, además de actitudes frías y despectivas con los compañeros de trabajo.

Para la dimensión de falta de realización personal en el trabajo sólo se encontró asociación significativa $(p \leq 0,05)$ con el tipo de Unidad Académica. El pertenecer a Enfermería representó un riesgo de 2 veces mayor para manifestar falta de realización personal en el trabajo. Esto demuestra que el personal de Enfermería se encuentra con una baja autoestima y desmotivación.

Se analizó la asociación entre las dimensiones del Work Engagement (vigor, absorción y dedicación) con las variables sociodemográficas así como laborales sin encontrar resultados significativos, a pesar de que el 99\% del personal académico mostró niveles altos en este constructo.

\section{DISCUSION}

Estudios sobre el estrés laboral crónico traducido en síndrome de Burnout han despertado gran interés ya que es un problema que no solo afecta la calidad de vida laboral de las personas, sino que repercute social y económicamente sin encontrar aún soluciones eficaces para dar respuesta y tratamiento a tan complejo problema. La prevalencia del Síndrome de Burnout fue del 63,5\% y por dimensiones la distribución fue la siguiente: 47,4\% con agotamiento emocional, 26,9\% con despersonalización y el 76,9\% mostró bajos niveles de realización personal en el trabajo. Dicha prevalencia coincide con Ángel Carlos Matía (2006) ${ }^{9}$ aunque es una muestra de médicos y enfermeras. En cambio hay prevalencias aún más altas de un $80 \%$ en docentes de primaria ${ }^{1}$ (Aldrete 2003) y secundaria (Aldrete 2008). ${ }^{10}$

En la docencia existe una mayor participación de la mujer, de ahí que en esta investigación el 62,8\% correspondió a este grupo, lo cual obedece a factores determinantes tales como: creciente urbanización, mayores niveles de educación, hogares dirigidos por mujeres y el elevado costo de vida, que han presionado la inserción de un mayor número de mujeres al campo de trabajo (Aldrete 2003). ${ }^{1}$ Respecto a esta variable se encontró una asociación significativa del sexo femenino con el agotamiento emocional, lo cual coincide con algunos estudios (Pando 2006, Pueyo 2000 y Hernández 2003). ${ }^{11-13}$

En cuanto a la edad, es una variable que se ha considerado como un indicador importante ya que la presencia de factores laborales, extralaborales y biológicos influyen para una mejor condición de madurez y estabilidad. En este estudio la edad promedio fue de 43,9 años, que coincide con la de otros estudios (Aldrete 2003, Oramas 2007 y Agudo 2005)..$^{1,14,15}$ El presente estudio mostró una relación significativa de la edad en aquellas personas de menos de 45 años con el agotamiento emocional, lo cual coincide con 
investigaciones que refieren que a mayor edad menor Burnout (Atance 1997 y Manzano 2001). ${ }^{16,17}$

Sobre la antigüedad se encontró que es un factor de riesgo para aquel profesional que cuenta con menos de cinco años y se asoció de manera significativa con la despersonalización, lo cual coincide con el estudio de Aranda ${ }^{18}$ (2004), donde el hecho de ser un docente de reciente ingreso crea sentimientos de incertidumbre laboral, trabajo poco valorado y reconocido, además de un mayor esfuerzo por sentirse parte de un grupo. De igual manera, Ponce Díz $^{19}$ (2005) expone que a mayor antigüedad el docente sufre menos agotamiento y despersonalización y sus niveles en la realización personal aumentan.

Respecto a la escolaridad, en este estudio el tener grado de doctor se asoció con el agotamiento emocional y la despersonalización, lo cual coincide con estudios donde se observa que el hecho de estar más preparado le otorga al académico nuevas posibilidades de promoción profesional así como diferentes exigencias que trascienden los límites del aula de clases, tales son las actividades correspondientes a perfiles de excelencia académica como el PROMEP (Programa de Mejoramiento del Profesorado) y SNI (Sistema Nacional de Investigadores) (Aldrete,2003). ${ }^{1}$

La literatura sobre el Work Engagement, Schaufeli y Bakker ${ }^{7}$ (2001) reportan que existe una relación entre la dimensión de agotamiento emocional y despersonalización con el vigor y dedicación, respectivamente, y que dicha relación se manifiesta como polo opuesto; esto es a mayores niveles en las dimensiones del Síndrome de Burnout, menores niveles en las dimensiones del Work Engagement, lo cual se contradice en este estudio, ya que ambos constructos resultaron con altos niveles en todas las dimensiones. En cuanto a los altos niveles de Work Engagement encontrados, se demostró que dentro del trabajo existen satisfactores en función de las creencias y valores, que se van desarrollando en el propio trabajo y que influirán de manera significativa para un mayor "enganche" laboral (Barraza 2008). ${ }^{20}$ También, como lo señala Arias $^{21}$ (2001), cuando existe un alto compromiso institucional, en este se encuentra implícito un deber moral adquirido a través de una amplia trayectoria y constante interacción hacia las personas y las instituciones, que se caracteriza por la creencia y aceptación de metas con total disposición para lograrlas a través de una intensa participación.

\section{CONCLUSIÓN}

Algunos estudios mencionan que el Síndrome de Burnout y el Work Engagement funcionan como polos opuestos, lo que indica que las variables de agotamiento emocional, despersonalización y falta de realización personal en el trabajo son significativamente negativas con el vigor, dedicación y absorción; inclusive se ha considerado que el agotamiento emocional y la despersonalización constituyen lo que se ha denominado el "corazón del burnout", de igual forma el vigor y la dedicación forman parte del "corazón del work engagment". Por lo tanto, serán dimensiones directamente opuestas: agotamiento vs vigor y despersonalización vs dedicación (Agudo 2005; GonzálezRomá 2006; García 2006). ${ }^{15,22,23}$

Como se puede observar, el presente trabajo contradice dicha teoría ya que se encontraron altos niveles para el agotamiento emocional, la despersonalización, el vigor, la dedicación y la absorción lo que indica que a pesar del estrés laboral crónico que pueda existir, también están presentes altos niveles de compromiso laboral, debido a que en el perfil docente se involucran aspectos humanistas como: responsabilidad asociada al compromiso, dedicación, cumplimiento, equidad, honestidad, tolerancia y prudencia; competencias y saberes que son el resultado no sólo de su formación profesional sino de aprendizajes realizados a lo largo de su vida, dentro y fuera de la escuela de acuerdo a sus contextos socioculturales y sus realidades educativas. 
1. Aldrete $M$, Pando $M$, Aranda $C$, Balcázar N. El Síndrome de Burnout en docentes de Educación Primaria de la Zona Metropolitana de Guadalajara. Investigación y Salud. 2003; 5(1):11-16.

2. Sandoval A. La equidad en la distribución de oportunidades de acceso a la educación media superior en México. Un estudio con base en los datos del EXANI-I Tesis de Maestria. México: Universidad Iberoamericana; 2005.

3. Gómez M y Bondjale T.: Sindrome de burnout o quemamiento de los profesionales. Prevención y tratamiento. La gestión del estrés. El cuidado de los cuidadores. En M. Gómez, editor. Cuidados paliativos e intervención psicosocial en enfermos terminales. Las Palmas: ICEPSS Eds.; 1994. p. 387-402,

4. Cifre E, Llorens S, Salanova M, Martinez IM. Salud psicosocial en profesores: repercusiones para la mejora en la gestión de los recursos humanos. Estudios Financieros. 2003; 50(247):153-247.

5. Schaufeli W, Salanova M, González-Roma V, Bakker AB. The measurement of engagement and burnout: $A$ two simple confirmatory factor analytic approach. J Happ Stud. 2002; (3):71-92,

6. Maslach C, Schaufeli WB, Leiter MP. Job Burnout. Annu Rev. Psychologist. 2001; (52):39-422,

7. Schaufeli WB, Bakker AB. Work and well-being: Towards a positive occupational health psychology. Gedrag in Organisatie. 2001;14:229-253,

8. Gil-Monte PR. Validez factorial de la adaptación al español del Maslach Burnout Inventory-General Survey. Salud Pública Méx. 2002; 44(1):33-40,

9. Matía AC, Cordero G, Mediavilla BJ, Pereda RM. Prevalencia de Burnout y variables asociadas en los médicos de atención primaria de Burgos. Atención Primaria. 2006; 38(1):55-59.

10. Aldrete MG, González J, Preciado ML. Factores psicosociales laborales y el síndrome de burnout en docentes de enseñanza media básica de la zona metropolitana de Guadalajara. Rev Chile Salud Pública. 2008;12(1):18-25.

11. Pando M, Castañeda J, Gómez $M$ et al. Factores psicosociales y sindrome de burnout en docentes de la Universidad del Valle de Atemejac, Guadalajara, México. Salud en Tabasco. 2006;12(3):523-559.

12. Pueyo RC. El sindrome de burnout o desgaste profesional. Madrid: JANO EMC; 2000,
13. Hernández JR. Estrés y burnout en profesionales de la salud de los niveles de primaria y secundaria de atención pública. Rev Cubana Salud Pública. 2003; 29(2):103-110,

14. Oramas $A$, Almirall $P$, Fernández I. Estrés laboral y el síndrome de burnout en docentes venezolanos. Salud de los Trabajadores. 2007;15:(21):71-87.

15. Agudo M. Burnout y engagement en profesores de primaria y secundaria. Jornadas de Fomento de la Investigación. Castelló de la Plana: Universitat JAUME I; 2005.

16. Atance JC. Aspectos epidemiológicos del síndrome de burnout en personal sanitario. Rev Esp Salud Pública [en línea] 1997; 71(3):293-303 [citado may 2014]. Disponible en: http://www.msc.es/biblioPublic/publicaciones/recursos_ propios/resp/revista.cdrom/VOL71/71_3_293,pdf.

17. Manzano G, Ramos F. Profesionales con alto riesgo de padecer burnout: enfermeras y secretarias. $2^{\circ}$ Congreso Virtual de Psiquiatria: Interpsiquis, feb 2001 [en línea]. Palmas de Mallorca: Intersalud; 2001; [citado abr 2014]. Disponible en internet: http://www. psiquiatria.com/psiquiatria/ revista/20/1243,

18. Aranda $C$, Pando M, Pérez MB. Apoyo social y síndrome de quemarse en el trabajo o burnout: una revisión. Investigación y Salud. 2004;14(1):79-87.

19. Ponce $C$, Bulnes $M$. El sindrome del quemado por estrés laboral asistencial en grupos de docentes universitarios. Revista IIPSI. 2005; 8(2):87-112,

20. Barraza MA, Acosta CM. Compromiso organizacional de los docentes de una institución de educación media superior. Innovación Educativa. 2008; $8(45): 21-35$.

21. Arias F. El compromiso personal hacia la organización y la intención de permanencia: algunos factores para su incremento. Rev Contad Admin. 2001; 200(1):5-12,

22. González-Romá V, Schaufeli WB, Bakker AB, Lloret S. Burnout and work engagement: Independent factor or opposite poles? J Vocat Behav. 200; (68):165-174.

23. Garcia M, Llorens S, Cifre E, Salanova M. Antecedentes afectivos de la autoeficacia docente: un modelo de relaciones estructurales. Rev Educ. 2006; 339: $387-400$, 